\title{
Characterization of Activated Carbon/SBA-15 with TEPA Modification
}

\author{
Zhifeng Lin \\ College of Environmental Science and Engineering, Guilin University of Technology, Guilin 541004, \\ China \\ 452296597@qq.com
}

\begin{abstract}
Keywords: TEPA, Activated carbon, SBA-15, Modification, Characterization.
Abstract. Activated carbon (AC), the KOH modified activated carbon (KAC) and SBA-15 before and after pore expansion (SBA-15k) were modified using tetraethylenepentamine (TEPA) as modifier to synthesize the adsorbents. The adsorbents were characterized by $\mathrm{N}_{2}$ adsorption-desorption and elemental analysis. The results showed that the pore size of SBA-15 materials were much larger than that of activated carbon. SBA-15k (P)-40 had the largest pore size which was mainly in the range of $10 \sim 20 \mathrm{~nm}$. KOH activation and SBA-15 reaming were beneficial to amino modification. In the same TEPA modification conditions, the actual TEPA loadings of SBA-15 materials were higher than those of activated carbons.
\end{abstract}

\section{Introduction}

The activated carbon is microporous materials, because of its huge specific surface area, highly developed pore structure, excellent surface chemical properties and adsorption performance, which has now been widely used in the fields of gas purification and recycling, wastewater treatment, chemical, pharmaceutical, food, defense, etc. [1,2]. SBA-15 type silicon oxide belongs to 2-D hexagonally mesoporous material with the characteristic such as large surface area, narrow pore size distribution and large pore size. Its thick pore wall improves hydrothermal and mechanical stability of the material, which is a potential adsorbent [3]. In general, the performance of microporous and mesoporous materials is associated with their surface physical and chemical properties. Increasing pore structure of materials by reaming or changing its chemical properties by adding amino groups can effectively improve the performance of materials. In recent years, more and more literatures reported the characterization of reaming or functional material and used it as a solid $\mathrm{CO}_{2}$ capture material [4-8]. But the amine modifications of activated carbon with reactivation and SBA-15 with reaming are seldom reported.

In this paper, TEPA was used as modifier to modify the activated carbon AC, KAC and SBA-15 before and after reaming. Then the differences of the structure and element concentration of the modified microporous and mesoporous materials were discussed to improve the adsorbents properties for the future use in the adsorption fields such as gas separation and heavy metals removal in water.

\section{Materials and Characterization}

The Synthesis and Modification of Activated Carbon and SBA-15 Materials. Activated carbon $\mathrm{AC}$ was prepared from bagasse using $\mathrm{ZnCl}_{2}$ as the activator. $\mathrm{KAC}$ was prepared from $\mathrm{AC}$ using $\mathrm{KOH}$ as the second activator.

KAC and AC were modified by using TEPA as modifier. Finally the modified materials with mass fraction of $10 \%, 20 \%, 30 \%, 40 \%$, and 50\% were prepared and recorded as AC-P-N and KAC-P-N. For example, AC-P-10 indicates that AC is modified with $10 \%$ TEPA.

The reaming SBA-15 was synthesized using nonionic surfactant P123 as template, tetraethoxysilane as the silicon source and TMB as expanding agent and was recorded as SBA-15k [9].

SBA-15 and SBA-15k were modified by impregnation method using TEPA as modifier. Finally the modified materials with mass fraction of $20 \%, 30 \%, 35 \%, 40 \%$, and $50 \%$ were prepared and recorded as SBA-15(P)-N and SBA-15k (P)-N. For example, SBA-15(P)-20 indicates that SBA-15 is modified with $20 \%$ TEPA [9]. 
Characterization of Materials. The pore structure of samples was determined by using NOVA2000e automatic surface analyzer (Quantachrome, USA) for nitrogen adsorption-desorption experiment in nitrogen atmosphere and at $-196^{\circ} \mathrm{C}$. The $\mathrm{C}, \mathrm{H}$ and $\mathrm{N}$ elements of samples were analyzed by using EA 2400 II elemental analyzer (Per-kin-Elmer, USA).

\section{Results and Discussion}

Analysis of Specific Surface Area and Pore Size of Adsorption Materials. The BET specific surface area, pore volume and pore size were calculated by the BJH method. The data is shown in Table 1. It can be seen from the Table 1, specific surface area and pore volume of AC-P-10 or KAC-P-10 are far larger than that of SBA-15k (P)-40 or SBA-15(P)-40.

Table 1 Physicochemical properties of adsorbents

\begin{tabular}{cccc}
\hline Samples & $\begin{array}{c}S_{\mathrm{BET}} / \\
\left(\mathrm{m}^{2} \cdot \mathrm{g}^{-1}\right)\end{array}$ & $\begin{array}{c}V_{\text {total }} / \\
\left(\mathrm{cm}^{3} \cdot \mathrm{g}^{-1}\right)\end{array}$ & $\begin{array}{c}D_{\mathrm{BET}} / \\
\mathrm{nm}\end{array}$ \\
\hline KAC-P-10 & 1235 & 0.85 & 2.74 \\
AC-P-10 & 766 & 0.37 & 1.52 \\
SBA-15k(P)-40 & 12.07 & 0.076 & 25.21 \\
SBA-15(P)-40 & 2.76 & 0.086 & 12.41 \\
\hline
\end{tabular}

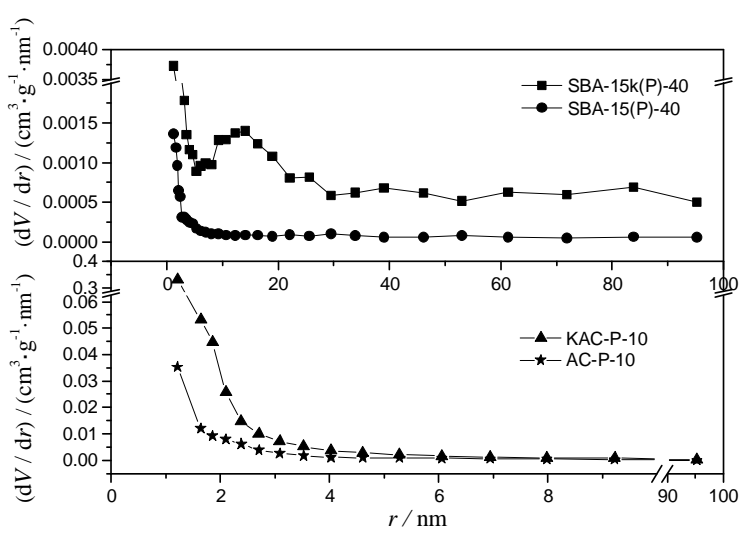

Fig. 1 Pore size distribution curve of mesopore of adsorbents

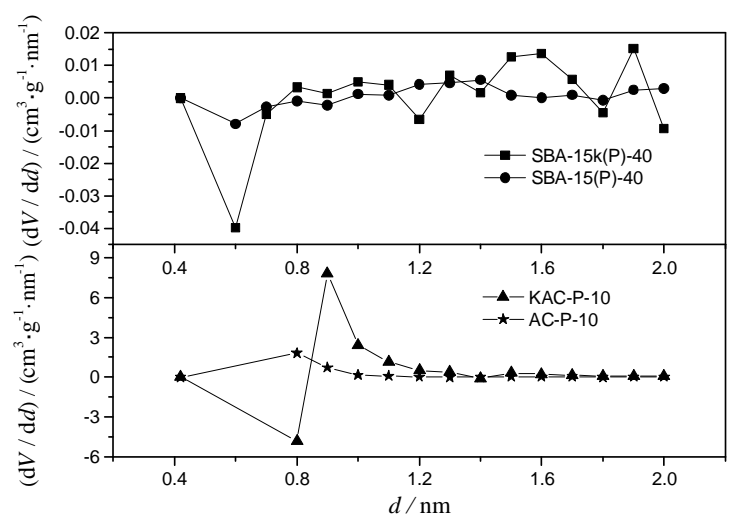

Fig. 2 Pore size distribution curve of micropore of adsorbents

Pore size distribution curve of mesopore and micropore of adsorbents are shown in Fig. 1 and Fig. 2 respectively. From Fig. 1 and Fig. 2 it can be seen that the pore size of SBA-15 materials are much larger than that of activated carbon. After reaming the SBA-15k (P)-40 owns largest pore size which 
was mainly distributed in $10 \sim 20 \mathrm{~nm}$. Its pore volume is $0.076 \mathrm{~cm}^{3} \cdot \mathrm{g}^{-1}$ and its pore size doubles that of SBA-15(P)-40 before reaming.

Elemental Analysis. The nitrogen content of adsorbents not only depends on the amine amount into the char, but also is related to the nature of the amine [10]. The elemental analysis results of materials are shown in Table 2. It can be seen from Table 2, the actual amine loading of various materials were lower than theoretical values. The actual amine loading of KAC-P-N and SBA-15k(P)-N were closer to the theoretical values than that of other materials, which indicated that amine was easier to be loaded on activated carbon after $\mathrm{KOH}$ activation or SBA-15 after reaming. This may be because that hole becomes larger after reaming for the access of amine, which led to the increasing of amino content. When the theoretical loading is same, the actual amine loading of SBA-15(P)-N is higher than that of AC-P-N. There are two reasons for this result. First, substance content of these two kinds of materials is not the same. SBA-15 is synthesized by P123 and TEOS through chemical reaction and in the pore of SBA-15 there are many sites where can be reached by amino. AC is prepared using bagasse as raw material at high temperature and its pore is relatively not so much that the active site to amino attachment. Second, dispersed phase is not the same. Amino load of SBA-15 or of AC is using absolute ethanol or ultra-pure water as dispersed phase respectively and the solubility of organic amine in two kinds of dispersed phase is not the same, which leads to the difference of actual amine loading. Although actual amine loading of KAC-P-N is slightly larger than that of AC-P-N, it is still less than that of SBA-15k (P)-N.

Table 2 Elemental analysis results of adsorbents

\begin{tabular}{ccccc}
\hline Samples & $\begin{array}{c}\text { Nitrogen ratio/ } \\
\%\end{array}$ & $\begin{array}{c}\text { Nitrogen content/ } \\
\left(\mathrm{mmol} \cdot \mathrm{g}^{-1}\right)\end{array}$ & $\begin{array}{c}\text { Amine content/ } \\
\left(\mathrm{mmol} \cdot \mathrm{g}^{-1}\right)\end{array}$ & $\begin{array}{c}\text { Actual loading/ } \\
\%\end{array}$ \\
\hline KAC-P-10 & 3.45 & 2.47 & 0.49 & 9.3 \\
KAC-P-20 & 6.19 & 4.43 & 0.89 & 16.7 \\
KAC-P-30 & 7.94 & 5.67 & 1.13 & 21.4 \\
KAC-P-40 & 11.03 & 7.88 & 1.58 & 29.5 \\
KAC-P-50 & 12.60 & 9.00 & 12.60 & 34.1 \\
AC-P-10 & 2.85 & 2.04 & 0.41 & 7.7 \\
AC-P-20 & 4.84 & 3.45 & 0.69 & 13.1 \\
AC-P-30 & 7.04 & 5.03 & 1,01 & 19.0 \\
AC-P-40 & 9.67 & 6.91 & 1.38 & 26.1 \\
AC-P-50 & 12.46 & 8.90 & 1.78 & 33.6 \\
SBA-15 k (P)-20 & 6.65 & 4.75 & 0.95 & 18.0 \\
SBA-15 k (P)-30 & 8.84 & 6.41 & 1.28 & 24.3 \\
SBA-15 k (P)-35 & 10.94 & 7.81 & 1.56 & 29.6 \\
SBA-15 k (P)-40 & 11.25 & 8.04 & 1.61 & 30.4 \\
SBA-15 (P)-20 & 6.23 & 4.45 & 0.89 & 16.8 \\
SBA-15 (P)-30 & 8.97 & 6.31 & 1.26 & 23.9 \\
SBA-15 (P)-35 & 9.64 & 6.89 & 1.37 & 26.1 \\
SBA-15 (P)-40 & 11.28 & 8.06 & 1.61 & 30.4 \\
\hline
\end{tabular}

\section{Conclusions}

This paper discusses the differences of structure and element concentrations between modified activated carbon and SBA-15 (including SBA-15k) through the methods of pore structure and element analysis. Both the active carbon materials and SBA-15 materials were modified by amine group and their actual amine loadings were less than the theoretical values. The pore size of SBA-15 materials was much larger than that of activated carbon. SBA-15k (P)-40 had the largest pore size which was mainly distributed in $10 \sim 20 \mathrm{~nm}$. $\mathrm{KOH}$ activation and SBA-15 reaming were beneficial to amino modification. In the same amino modification conditions, the actual amino loadings of SBA-15 materials were higher than those of activated carbons. 


\section{Acknowledgements}

The author thanks the supports of National Natural Science Foundation of China (No.51566003) and National Natural Science Foundation of Guangxi (No.2015GXNSFAA139231).

\section{References}

[1] S.J. Zhang, H.M. Feng, J.P. Wang and H.Q. Yu: Journal of Colloid and Interface Science, Vol. 321 (2008), p. 96-102.

[2] M.L. Gray, Y. Soong, K.J. Champagne, J. Baltrus, R.W.S. Jr, P. Toochinda and S.S.C. Chuang: Separation and Purification Technology, Vol. 35 (2004), p. 31-36.

[3] J.W. Wei, J.H. Wang, S.S. Zhao and L.L. Geng: Journal of Inorganic Materials, Vol. 31 (2016), p. 449-453 (In Chinese).

[4] C. Chen and Y.X. Dong: Journal of Xinyang Normal University Natural Science Edition, Vol. 28 (2015), p. 102-105.

[5] M.B. Yue, Y. Chun, Y. Cao, X. Dong and J.H. Zhu: Advanced Functional Materials, Vol. 16 (2006), p. 1717-1722.

[6] A. Sayari: Angewandte Chemie International Edition, Vol. 39 (2000), p. 2920-2922.

[7] H.Y. Huang, R.T. Yang, D. Chinn and C.L. Munson: Industrial \& Engineering Chemistry Research, Vol. 42 (2003), p. 2427-2433.

[8] Y.L. Ji, K. Liang and J.H. Gong: China Sciencepaper, Vol. 8 (2013), p. 192-195 (In Chinese).

[9] J.W. Wei, K.K. He, L.S. Meng and L. Liao: Chinese Journal of Environmental Engineering, Vol. 9 (2015), p. 4447-4452 (In Chinese).

[10] M.G. Plaza, C. Pevida, A. Arenillas, F. Rubiera and J.J. Pis: Fuel, Vol. 86 (2007), p. 2204-2212. 